\title{
Endovascular Stent Graft Exclusion of an latrogenic Tracheoinnominate Fistula after Unsuccessful Surgical Repair
}

\author{
Andrew Woerner ${ }^{1}$ Kevin S. H. Koo ${ }^{2}$ Eric J. Monroe ${ }^{2}$ \\ ${ }^{1}$ Loyola University Chicago Stritch School of Medicine, Chicago, \\ Illinois, United States \\ ${ }^{2}$ Department of Radiology, Section of Interventional Radiology, \\ Seattle Children's Hospital and University of Washington, Seattle, \\ Washington, United States \\ J Clin Interv Radiol ISVIR 2018;2:106-109
}

\begin{abstract}
Address for correspondence Eric J. Monroe, MD, Department of Radiology, Seattle Children's Hospital, 4800 Sand Point Way NE, M/S R-5417, Seattle, WA 98105, United States (e-mail: eric.monroe@seattlechildrens.org).
\end{abstract}
Abstract
Keywords
- pseudoaneurysm
- innominate artery
- stent

A 10-year-old girl with metachromatic leukodystrophy developed a tracheoinnominate artery fistula and massive hemoptysis following tracheostomy, initially requiring open surgical repair. Massive hemoptysis recurred at 1 month due to suspected pericardial patch dehiscence. The authors herein describe the subsequent emergent treatment by stent graft exclusion and review the existing literature surrounding this rare and potentially fatal condition.

\section{Introduction}

A tracheoinnominate artery fistula (TIF) is a rare complication following tracheostomy. The mortality rate of this complication is $100 \%$ if untreated, making prompt diagnosis and management critical to ensure survival. ${ }^{1}$ Interventions using an open repair strategy have even shown mortality rates approaching $50 \% .^{2}$ Minimally invasive approaches to treatment may prove to be a safer alternative to open repair; however, there is a paucity of literature on this topic, especially in the pediatric setting. Here, we describe a case of TIF in a 10 -year-old girl treated with endovascular stent graft placement.

\section{Case Summary}

A 10-year-old girl with metachromatic leukodystrophy and epilepsy underwent tracheostomy at an outside institution due to chronic respiratory failure. One month after the tracheostomy creation, she presented acutely at the outside institution with large volume hemoptysis within and around her tracheostomy. Imaging at that time confirmed a low tracheostomy in close approximation with the innominate artery ( - Fig. 1), presumably leading to erosion of the artery with bleeding through pressure necrosis of the anterior wall of the trachea. Bleeding was temporized by a cuffed endotracheal tube and a pressure dressing at the neck.

received

September 9, 2017

accepted after revision

February 1, 2018

published online

June 28, 2018
The patient was transferred emergently to our institution for surgical correction. The great vessels and trachea were exposed via median sternotomy. A $2.5-\mathrm{mm}$ defect was identified in the cephalad aspect of the innominate artery and repaired using sutured pericardial pledgets. The tracheotomy was exposed and sealed with sutured pericardial pledget. A sternocleidomastoid muscle flap was then mobilized and interposed between the repaired innominate artery and tracheotomy. She did well immediately postprocedure and underwent a new, more superiorly placed tracheostomy placement on hospital day 17 . Her tracheostomy was changed and evaluated on hospital day 24 . She continued to recover well with no recurrent hemoptysis and was transferred to her home state for continued management 30 days postsurgical repair.

Upon arrival to the outside hospital, the patient had recurrent massive hemoptysis via her tracheostomy. After approximately $250 \mathrm{~mL}$ of expectorated blood loss, bleeding was controlled with a cuffed endotracheal tube. Her presentation was notable for acidosis to 7.01 on arterial blood gas as well as acute blood loss requiring transfusion. She was immediately transferred back to our institution. Arterial-phase computed tomography (CTA) confirmed the presence of an innominate artery pseudoaneurysm (-Fig. 2), and the patient was taken emergently to the angiography suite (now day 31 since original TIF repair). A long $8 \mathrm{~F} 65-\mathrm{cm}$ vascular
License terms

ISSN 2457-0214. Radiology 

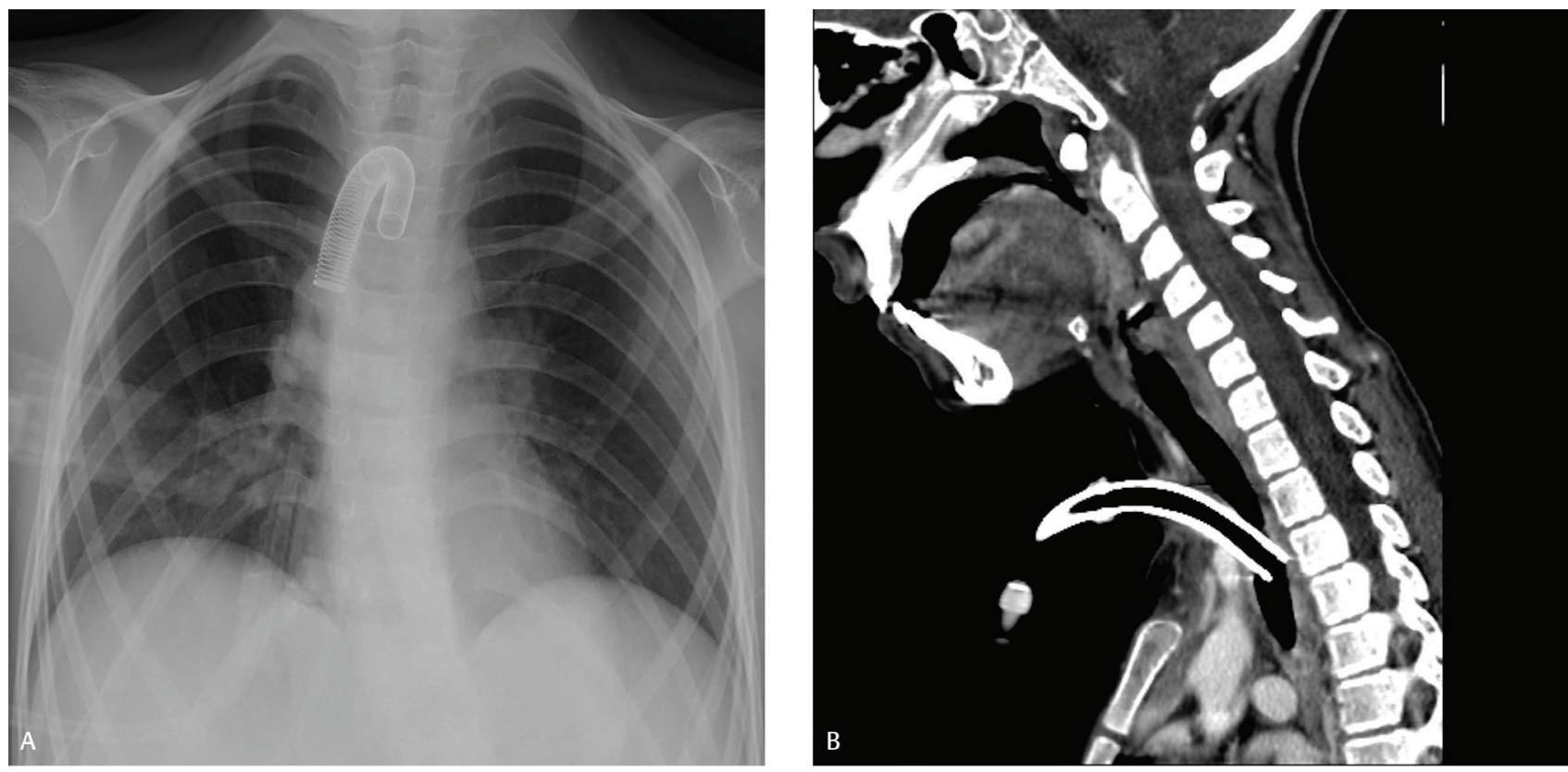

Fig. 1 Semi-upright chest radiograph (A) and sagittal CTA (B) depicting the low tracheostomy placement abutting the innominate artery.

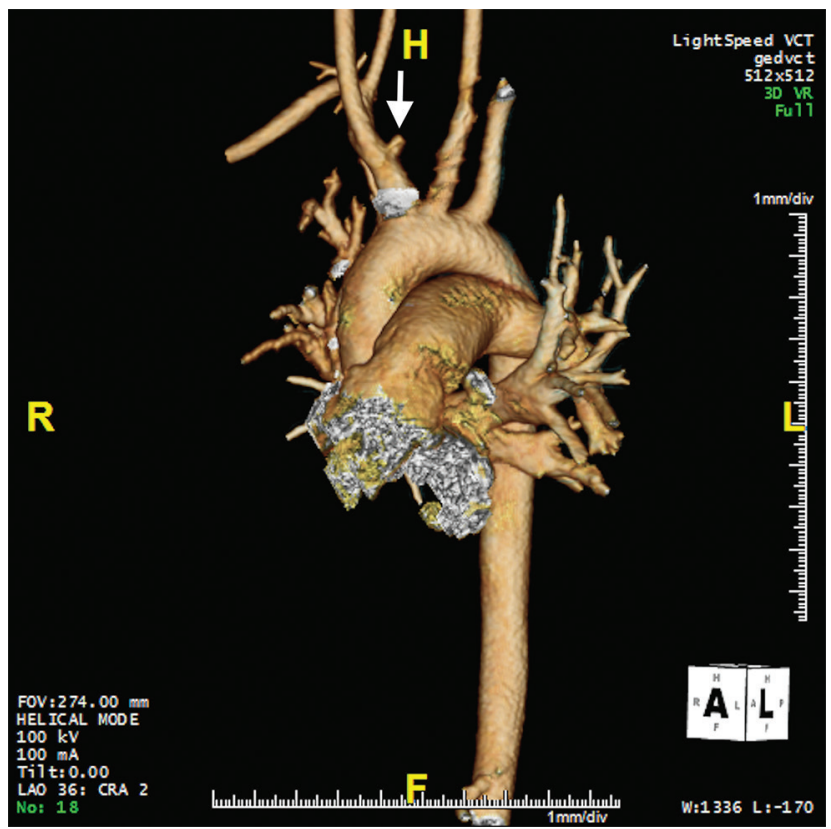

Fig. 2 Three-dimensional surface rendering of CTA displaying an innominate artery pseudoaneurysm (arrow).

sheath (Pinnacle Destination, Terumo Medical) was placed following right femoral artery access. A $4 \mathrm{~F}$ angled catheter and 0.035-in hydrophilic wire were used to select the innominate and then the right subclavian artery. Innominate artery angiograms were acquired in multiple projections, confirming a 2- $\times 3-\times 3-\mathrm{mm}$ pseudoaneurysm of the superior surface of the innominate artery ( - Fig. $\mathbf{3}$ ).

The sheath was advanced into the right subclavian artery with its dilator, and the dilator was then removed. A $7 \times 19 \mathrm{~mm}$ balloon expandable stent graft (Viabahn VBX, W.L. Gore \& Associates) was positioned in the innominate artery ( - Fig. 4) and deployed to nominal diameter. An $8 \times 20 \mathrm{~mm}$

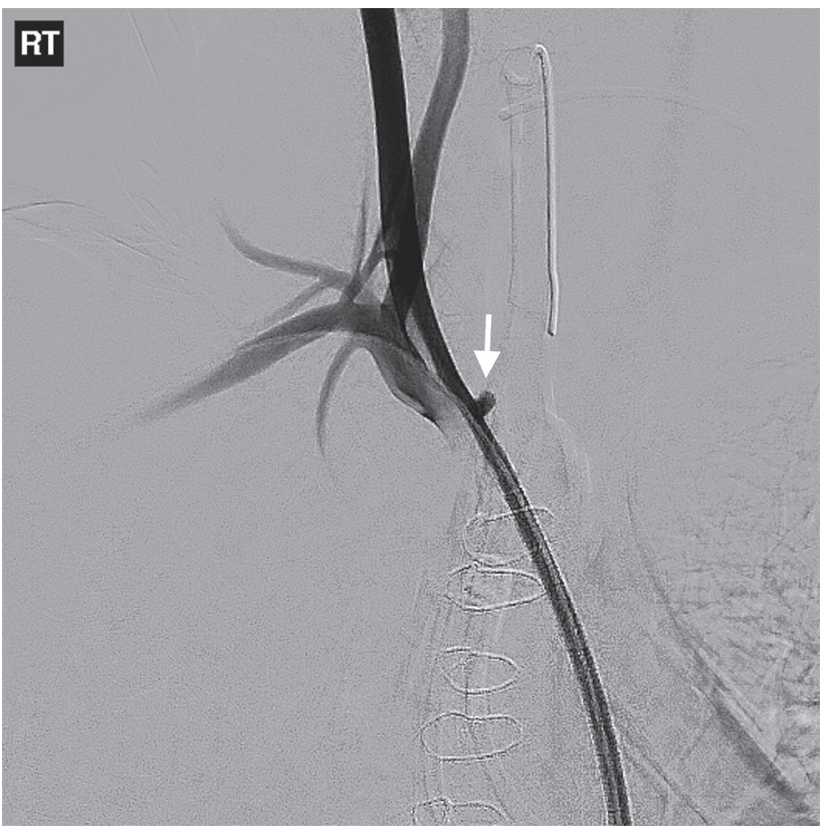

Fig. 3 Digital subtraction angiogram depicting a pseudoaneurysm on the superior surface of the innominate artery, $1 \mathrm{~mm}$ proximal to the origin of the right common carotid artery, without angiographic extravasation (arrow).

balloon was introduced and inflated across the proximal aspects of the stent graft for improved apposition to the arterial wall. Completion angiogram confirmed complete exclusion of the pseudoaneurysm with patent great vessels and no endoleak (-Fig. 5).

Following stent graft deployment, endotracheal tube cuff was deflated and hemostasis was confirmed. The patient tolerated the procedure well and was transferred back to the intensive care unit (ICU) in stable condition. Although the patient displayed no clinical signs of a mycotic component to 


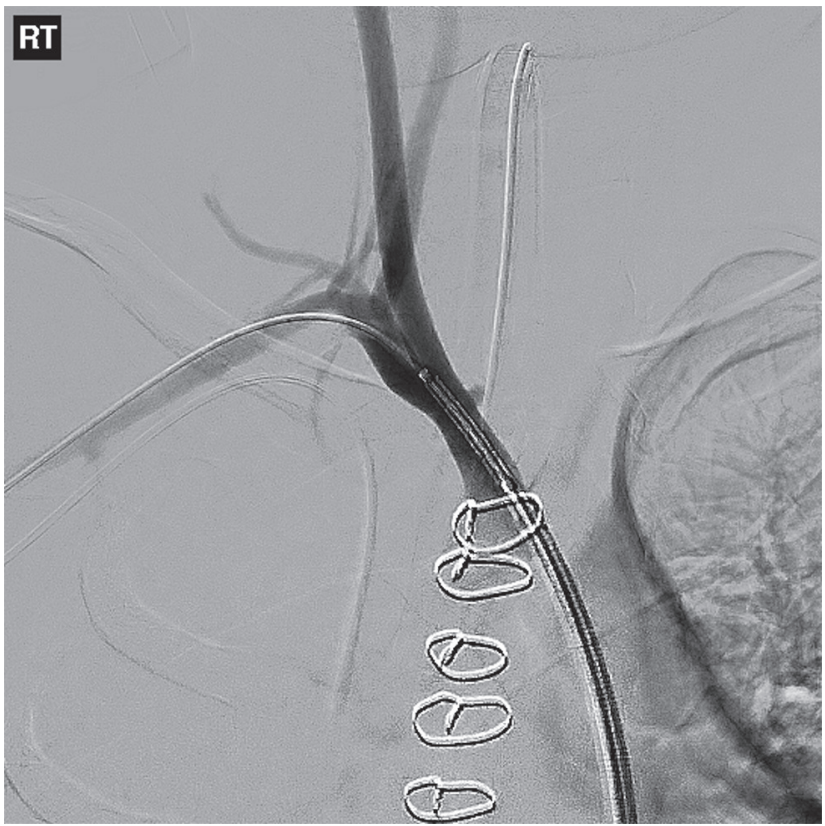

Fig. 4 Digital subtraction angiogram of the innominate artery for stent graft positioning prior to deployment.

her recurrent pseudoaneurysm, broad-spectrum intravenous antibiotics were initiated periprocedurally and continued for 72 hours with piperacillin/tazobactam 2,025 mg intravenous every 8 hours. A daily aspirin was initiated on the first postprocedure day for prophylaxis against stent graft thrombosis and cerebrovascular complications. She remained in the ICU while weaning her ventilator settings and sedation, ultimately returning to her baseline and being discharged home 2 weeks status post stent-graft placement. At the time of preparing this article, 4 months since placement, she has recovered well with no further episodes of hemoptysis. She is followed expectantly with a planned surveillance CTA 1 year from the procedure. Aspirin will continue indefinitely unless contraindicated.

\section{Discussion}

Tracheoinnominate artery fistula formation is a life-threatening complication of tracheostomy. The formation rate remains low in the reported literature (0.2-0.7\%); however, the mortality rate is still significant. ${ }^{3}$ In a review of 1,130 cases of tracheostomy placement in children, Goldenberg et al found only 2 reported instances of TIF formation, both of which were fatal. ${ }^{3}$ Survival without treatment of the TIF has never been reported.

Tracheoinnominate artery fistulas predominantly occur between the first and second weeks after tracheostomy, most commonly due to erosion of the innominate artery vessel by the inferior neck of a rigid tracheostomy tube that is placed too low. Also, an anomalously high innominate artery, more common in thin and younger patients, places patients at higher risk. Because of this, tracheostomy tubes should not be placed below the third tracheal ring. Additional factors that may predispose individuals to TIF formation include overinflating of cuffs, tracheostomy tubes with adjustable flanges, and positive airway pressure. ${ }^{4}$

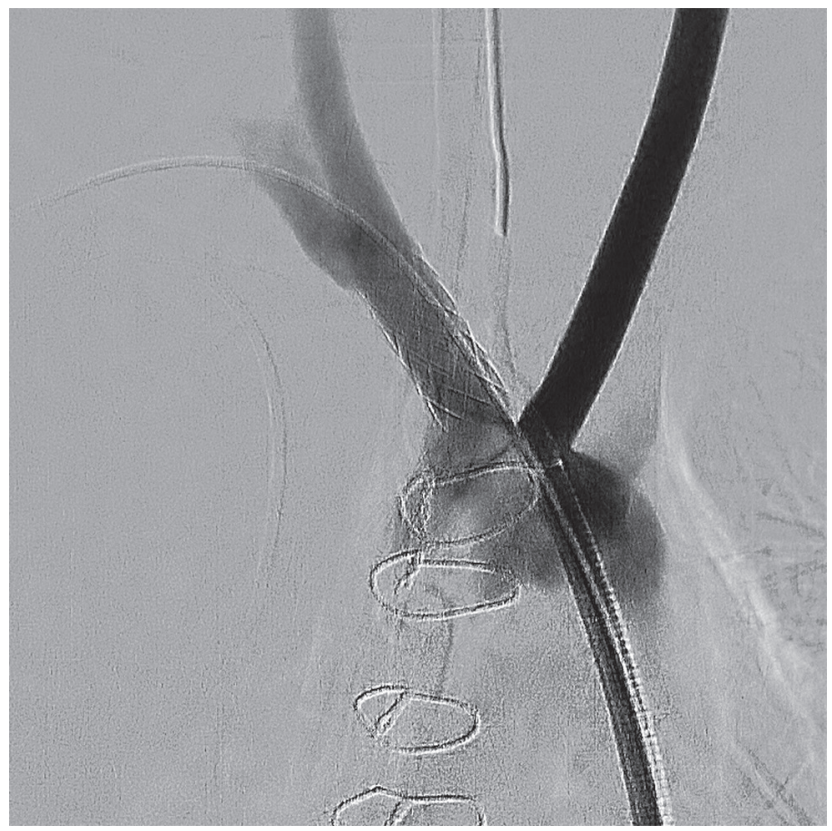

Fig. 5 Digital subtraction angiogram of the innominate artery following stent graft deployment, confirming pseudoaneurysm exclusion, great vessel patency, and no endoleak.

Several classic clinical features should raise suspicion for a TIF. Any peristomal bleeding and hemoptysis warrant clinical evaluation. Bleeding witnessed within 48 hours of tracheostomy is typically not associated with a TIF, rather from a possible traumatic puncture of local venous structures, a coagulopathy, or from traumatic tracheal secretion suctioning. On the other hand, bleeding that occurs between 3 days and 6 weeks after tracheostomy is a TIF until proven otherwise. A sentinel small peristomal bleed that spontaneously resolves occasionally precedes more massive hemoptysis. A pulsating tracheostomy suggests close proximity to a major artery and should raise suspicion for TIF. Placement of a cuffed tracheostomy tube is a critical temporizing measure and helps confirm the diagnosis if hemoptysis ceases.

Historically, open surgical repair has been the treatment of choice for TIF. Unfortunately, mortality rates for open repair exceed $50 \%$ in previous reviews, and long-term survival is poor with only 8 to $56 \%$ of patients living beyond 2 months. ${ }^{2}$ Given the poor surgical outcomes, investigation of alternative treatment is warranted. Operators taking an endovascular approach may have concerns for the use of synthetic materials in the proximity of a likely infected area. In addressing this issue, Sorial et al assembled outcomes of various endovascular attempts to treat TIF, and of the five cases presented, none had any reported infectious complications. ${ }^{1}$

A literature review yielded 13 published cases of TIF treated endovascularly. Only four of these cases have been in children. Takasaki et al reported a case of a 9-year-old boy with TIF successfully treated by embolization of the innominate artery. ${ }^{4}$ The case reported by Vianello and colleagues is the first known successful stent grafting in a pediatric case. ${ }^{5}$ Further reports have continued to use stent grafting as the treatment choice in children with success and known stable outcomes at several weeks to 24 months after the procedure. ${ }^{6-8}$ 
Endovascular treatment was pursued after the patient recurred postsurgical repair as repeat surgical exploration was considered to carry unacceptable risks. The endovascular approach allows rapid control of bleeding while avoiding the morbidity of a median sternotomy, and we speculate a more favorable course had the patient been treated primarily in this manner. Meanwhile, we acknowledge limitations in the assessment of treatment durability and a generalized lack of available literature on long-term outcomes. Furthermore, airway continuity with the vascular injury introduces the potential for stent graft infection and associated complications that could necessitate surgical correction. Our review identified no cases of endovascular material infection, but the sparse amount of available data is insufficient to dismiss this possibility and we recommend broad-spectrum periprocedural antibiosis until further guidance becomes available. In the absence of antiplatelet guidance specific to stenting for this condition, we have extrapolated our plan for indefinite daily aspirin therapy from the iliac artery covered stent literature.

TIFs present a formidable treatment challenge. A limited but growing body of literature supports endovascular repair. Here, we have presented a case of a TIF in a 10-year-old girl treated with endovascular stent graft placement following a recurrence after surgical treatment. The procedure was uncomplicated, successful, and well tolerated, adding to the evidence that endovascular stent grafting of the innominate artery can be a safe treatment option for TIF in the pediatric population.

\section{Conflicts of Interest}

The authors report no conflicts of interest.

\section{Ethical Approval}

All procedures performed in studies involving human participants were in accordance with the ethical standards of the institutional and/or national research committee and with the 1964 Declaration of Helsinki and its later amendments or comparable ethical standards.

\section{Informed Consent}

Does not apply.

\section{References}

1 Sorial E, Valentino J, Given CA, Endean ED, Minion DJ. The emergency use of endografts in the carotid circulation to control hemorrhage in potentially contaminated fields. J Vasc Surg 2007;46(4):792-798

2 Brewster DC, Moncure AC, Darling RC, Ambrosino JJ, Abbott WM. Innominate artery lesions: problems encountered and lessons learned. J Vasc Surg 1985;2(1):99-112

3 Goldenberg D, Ari EG, Golz A, Danino J, Netzer A, Joachims HZ. Tracheotomy complications: a retrospective study of 1130 cases. Otolaryngol Head Neck Surg 2000;123(4):495-500

4 Takasaki K, Enatsu K, Nakayama M, Uchida T, Takahashi H. A case with tracheo-innominate artery fistula. Successful management of endovascular embolization of innominate artery. Auris Nasus Larynx 2005;32(2):195-198

5 Vianello A, Ragazzi R, Mirri L, Arcaro G, Cutrone C, Fittà C. Tracheoinnominate fistula in a Duchenne muscular dystrophy patient: successful management with an endovascular stent. Neuromuscul Disord 2005;15(8):569-571

6 Joshi AS, Tanna N, Elmaraghy CA, et al. Nonsurgical treatment of tracheoinnominate fistula in the pediatric population. Arch Otolaryngol Head Neck Surg 2007;133(3):294-296

7 Cavalcante RN, Motta-Leal-Filho JM, Maciel MJS, Carnevale FC. Tracheoinnominate fistula: endovascular treatment with a stent graft in a 4-year-old child. Ann Vasc Surg 2016;31:206. e9-206.e12

8 Bae M-H, Lee Y-J, Nam SO, Kim H-Y, Kim CW, Kim YM. Endovascular stenting of tracheoinnominate fistula after tracheostomy in a 14-year-old boy. Korean J Pediatr 2016;59 (Suppl 1): S76-S79 